УДК 519.618

DOI: $10.21779 / 2542-0321-2018-33-3-73-79$

\title{
А.3. Якубов
}

\section{Применение потоково-графовых методов в оптимизации расписания}

Дагестанский государственный университет; Россия, 367001, 2. Махачкала, ул. М. Гаджиева,43a; yakubovaz@mail.ru

Оптимизация расписания учебных занятий сформулирована в виде задачи дефрагментации матрицы: для матрицы из семи столбцов, каждый из которых содержит, кроме нулей, некоторую перестановку первых $n$ натуральных чисел, рассматривается вопрос существования матрицы тех же размеров, сохраняющей множество элементов исходной матрицы в каждом столбце и каждой строке таким образом, что в каждой строке все ненулевые элементы расположены слитно. Благодаря применению потоково-графовых методов найдены необходимые и достаточные условия дефрагментации матрицы в некоторых частных случаях.

Ключевые слова: оптимизаџия расписания, дефрагментация матрии, потоки в сетях, паросочетания.

\section{Введение}

В статье рассматривается задача дискретной оптимизации - дефрагментации матриц различных классов, т. е. непрерывного расположения ненулевых элементов в каждой строке матрицы, сохраняющей множество элементов в каждой линии матрицы. Сложность исследования приведенной задачи является результатом труднорешаемости [2] соответствующих математических моделей. В связи с этим в статье рассматривается частный случай, допускающий решение за полиномиальное время.

В качестве модели, объединяющей в себе характерные черты сформулированных и близких к ним задач, в заключении данной работы рассматривается задача оптимизациии расписания учебных занятий по критерию уменьшения окон преподавателей.

\section{Формулировка задачи}

1) $I_{n}=\{1,2,3, \ldots, \mathrm{n}\} ; I_{n, k}-k$-элементное множество $\{1,2,3, \ldots, \mathrm{n}, 0,0, \ldots, 0\}$.

2) $P^{[k, m, n]}$ - матрицы размеров $k \times m$, где каждый столбец содержит в точности один экземпляр каждого элемента из $I_{n, k}$.

3) $P_{s}^{[k, m, n]}$ набор матриц из класса $P^{[k, m, n]}$, для каждой из которых количество ненулевых элементов в каждой строке представлено элементом множества s.

4) Множество строк в матрице $M$, каждая из которых содержит в точности $q$ ненулевые элементы, будем обозначать через $M^{(q)}$.

Сформулируем задачу дефрагментации матрицы в виде задачи распознавания свойств [2, с. 27].

Задача «Дефрагментация матрицы» (ДМ).

Условие. $M$ - матрица класса $P^{[k, m, n]}, k, m, n$ - целые положительные числа.

Bonpoc. Существует ли матрица $M^{*} k \times m$ для матрицы $M$, удовлетворяющая усло- 
виям:

- каждая строка и каждый столбец искомой матрицы $M^{*}$ сохраняют количество экземпляров каждого числа таким же, что и в соответствующей строке и столбце исходной матрицы $M$;

- выполняется условие непрерывного размещения ненулевых элементов в каждой строке матрицы $M^{*}$.

Нетривиальность поставленной задачи показывает рис. 1. Для матрицы $A$, очевидно, не существует дефрагментированного варианта. Матрица $B$ дефрагментируема, дефрагментированный вариант представлен рядом.

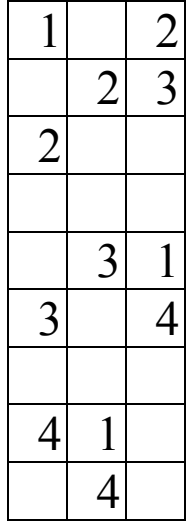

A

\begin{tabular}{|l|l|l|l|l|l|l|}
\hline 1 & & 3 & & & 2 & \\
\hline & & 4 & 1 & 2 & 3 & \\
\hline 2 & 3 & & 5 & & & 4 \\
\hline & & & & 3 & 1 & 2 \\
\hline 3 & 4 & & 2 & 1 & & 5 \\
\hline & & & & 4 & 5 & 3 \\
\hline 4 & 5 & 1 & 3 & & & \\
\hline & 2 & & & 5 & & 1 \\
\hline 5 & 1 & 2 & & & & \\
\hline & & & 4 & & & \\
\hline & & 5 & & & 4 & \\
\hline
\end{tabular}

B

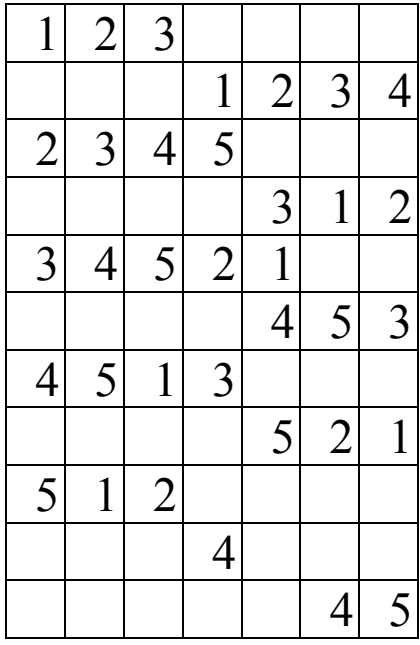

$\mathrm{C}$

Рис. 1. Матрица А не допускает дефрагментацию. Матрица В дефрагментируема, $\mathrm{C}$ - дефрагментированный вариант B

Необходимые и достаточные условия дефрагментации класса $P^{[k, m, n]}$ при $4 \leq m \leq 6$ приведены в работах $[7,8]$. В параграфах 2-3 данной статьи рассматриваются условия дефрагментации матриц класса $p^{[k, 7, n]}$. Критерий дефрагментации матрицы из 7 столбцов, удовлетворяющей вышеприведенным условиям, сформулирован в терминах потоков в транспортных сетях [6].

\section{Вычислительная сложность задачи ДМ-01}

Для положительного ответа на вопрос задачи ДМ, сформулированной выше, необходимо иметь положительный ответ на вопрос следующей задачи.

Задача «Дефрагментация матрицы из 0-1» (ДМ-01).

Условие. Дана матрица $M k \times m$, каждый столбец которой содержит $n$ единиц и $k-n$ нулей. Также даны натуральные $k, m, n(k \geq n)$.

Bonpoc. Существует ли для матрицы $M$ матрица $M^{*} k \times m$, где в каждой линии матрицы сохранено соотношение нулей и единиц, причем единицы в каждой строке следуют непрерывно?

В [8] показано, что задача ДM-01 является NP-полной даже при $n=2$. Указано полиномиальное сведение известной $N P$-полной задачи «Разбиение» к задаче «ДM-01», сформулированной для случая $n=2$. 


\section{Дефрагментация матриц класса $p_{\{2,5,7\}}^{[k, 7, n]}$}

Необходимо проверить существование для исходной матрицы $M$ класса $p_{\{2,5,7\}}^{[k, 7, n]}$, матрицы $M^{*} k \times m$, удовлетворяющей вышеперечисленным условиям, считая, что все ненулевые элементы в каждой строке попарно различны.

Для исследования условий дефрагментации матрицы используем потоки в транспортной сети. Обозначения:

$T(M)$ - транспортная сеть для матрицы $M$;

$[a, b], a-$ нижнее потоковое ограничение, $b$ - верхнее потоковое ограничение.

Вершинь:

$T_{1}$ - источник, $T_{2}$ - сток;

элементам множества $\{1,2, \ldots n\}$ матрицы $M$ соотнесены $n$ вершин $x_{1}, x_{2}, \ldots, x_{n}$ транспортной сети;

строкам множеств $M^{(5)} u M^{(7)}$ функцией $\eta$ последовательно соотнесены $\chi$ вершин $y_{1}, y_{2}, \ldots, y_{\chi}$ транспортной сети, определенной на множестве этих вершин,

$\eta:\left\{y_{1}, y_{2}, \ldots, y_{\chi}\right\} \rightarrow M^{(5)} \cup M^{(7)}$;

$\chi$ представляет собой количество строк, где число ненулевых элементов принадлежит множеству $\{5,7\}$, т. е. $\chi \equiv k-\left|M^{(2)}\right|$.

Дуги:

к каждой из вершин $x_{s}$ от источника $T_{l}$ проведена дуга с потоковыми ограничениями $[3,3]$;

к вершине $y_{t}(t=1,2, \ldots, \chi)$ от вершины $x_{s}(s=1,2, \ldots, n)$ дуга проведена только в том случае, если строка $M\left(y_{t}\right)$ содержит число $s$, при этом потоковые ограничения по дуге $\left(x_{s}, y_{t}\right)$ равны $[0,1]$;

к стоку $T_{2}$ от каждой вершины $y_{t}$ проведена дуга с ограничениями $[3,3]$.

Таким образом, транспортная сеть $T(M)$ построена.

Из определения транспортной сети $T(M)$ вытекает следующее свойство: при существовании допустимого потока в $T(M)$ его величина будет равна $3 \times n$. В [11] доказана следующая теорема:

Теорема 3.1. Для дефрагментации матрицы $M$ необходимо и достаточно существование допустимого потока в транспортной сети $T(M)$.

Из доказательства теоремы выделим вычислительную схему дефрагментации матрицы $M$.

По матрице $M$, представленной на рис. 2 , приведена транспортная сеть $T(M)$, построенная на рис. 3. Двудольный граф $G=(X \cup Y, E)$, построенный на дугах $E$ сети $T(M)$, по которым протекает поток величины 1 , представлен на рис. 4 , там же выделено одно совершенное паросочетание: $\left\{\left(\mathrm{x}_{1}, \mathrm{y}_{1}\right),\left(\mathrm{x}_{2}, \mathrm{y}_{3}\right),\left(\mathrm{x}_{3}, \mathrm{y}_{5}\right),\left(\mathrm{x}_{4}, \mathrm{y}_{2}\right),\left(\mathrm{x}_{5}, \mathrm{y}_{6}\right),\left(\mathrm{x}_{6}, \mathrm{y}_{3}\right)\left(\mathrm{x}_{7}, \mathrm{y}_{7}\right)\right\}$.

После удаления этого паросочетания остовный подграф степени 2 (в нашем случае на рис. 5 - гамильтонов цикл) допускает очевидное расслоение на два совершенных паросочетания: $\left.\left\{\left(\mathrm{x}_{1}, \mathrm{y}_{4}\right),\left(\mathrm{x}_{2}, \mathrm{y}_{1}\right),\left(\mathrm{x}_{3}, \mathrm{y}_{1}\right),\left(\mathrm{x}_{4}, \mathrm{y}_{4}\right),\left(\mathrm{x}_{5}, \mathrm{y}_{2}\right),\left(\mathrm{x}_{6}, \mathrm{y}_{3}\right)\left(\mathrm{x}_{7}, \mathrm{y}_{2}\right)\right)\right\}$ и $\left\{\left(\mathrm{x}_{1}, \mathrm{y}_{6}\right),\left(\mathrm{x}_{2}\right.\right.$, $\left.\left.\mathrm{y}_{5}\right),\left(\mathrm{x}_{3}, \mathrm{y}_{7}\right),\left(\mathrm{x}_{4}, \mathrm{y}_{6}\right),\left(\mathrm{x}_{5}, \mathrm{y}_{5}\right),\left(\mathrm{x}_{6}, \mathrm{y}_{7}\right)\left(\mathrm{x}_{7}, \mathrm{y}_{3}\right)\right\}$.

Отсюда видно, что $\mathrm{X}_{1}=\mathrm{X}_{2}=\mathrm{X}_{3}=\left\{\mathrm{x}_{1}, \mathrm{x}_{2}, \mathrm{x}_{3}, \mathrm{x}_{4}, \mathrm{x}_{5}, \mathrm{x}_{6}, \mathrm{x}_{7}\right\}$.

Выделим также два множества: $\mathrm{W}_{1}, \mathrm{~W}_{2}$, $\mathrm{W}_{1}=\{(4,5),(2,6),(3,4),(5,1),(2,3),(7,1),(7,6)\}$, $\mathrm{W}_{2}=\{(6,7),(3,4),(1,7),(2,3),(5,2),(5,1),(4,6)\}$. 


\begin{tabular}{|c|c|c|c|c|c|c|c|}
\hline & 1 & 2 & 3 & 4 & 5 & 6 & 7 \\
\hline 1 & 1 & 2 & 3 & 4 & 5 & 6 & 7 \\
\hline 2 & 2 & 0 & 4 & 5 & 6 & 7 & 0 \\
\hline 3 & 0 & 3 & 0 & 0 & 0 & 0 & 4 \\
\hline 4 & 6 & 7 & 0 & 3 & 4 & 2 & 0 \\
\hline 5 & 0 & 0 & 5 & 0 & 0 & 0 & 1 \\
\hline 6 & 0 & 4 & 1 & 0 & 2 & 3 & 6 \\
\hline 7 & 7 & 0 & 0 & 1 & 0 & 0 & 0 \\
\hline 8 & 3 & 1 & 0 & 0 & 7 & 5 & 2 \\
\hline 9 & 0 & 0 & 6 & 7 & 0 & 0 & 0 \\
\hline 10 & 4 & 0 & 0 & 2 & 3 & 1 & 5 \\
\hline 11 & 0 & 5 & 2 & 0 & 0 & 0 & 0 \\
\hline 12 & 5 & 0 & 7 & 6 & 1 & 0 & 3 \\
\hline 12 & 0 & 6 & 0 & 0 & 0 & 4 & 0 \\
\hline
\end{tabular}

Рис. 2. Исходная матрица М класса $\quad, \mathrm{k}=13, \mathrm{~m}=7, \mathrm{n}=7, \mathrm{~s}=\{2,5,7\}$

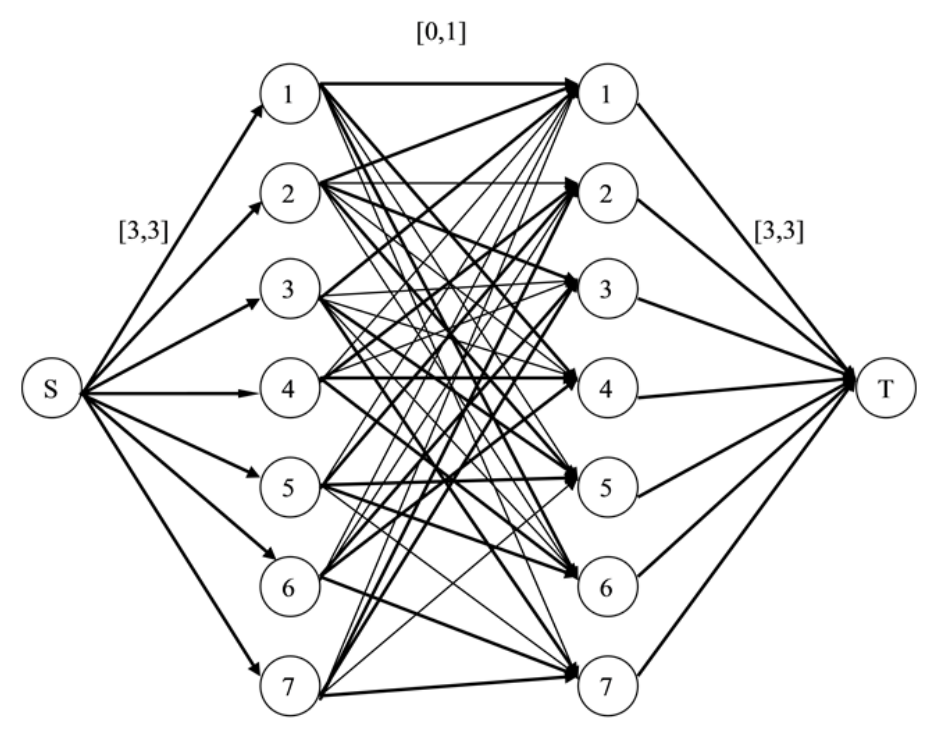

Рис. 3. Транспортная сеть Т(М), построенная по матрице М на рис. 2. Потоковые ограничения дуг указаны в квадратных скобках 


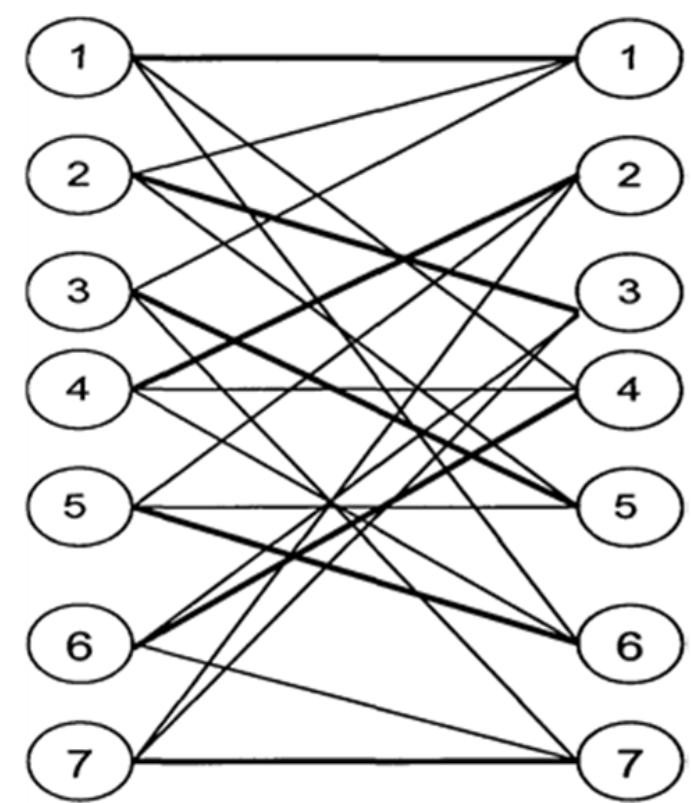

Рис. 4. Двудольный граф $\mathrm{G}=(\mathrm{X} \cup \mathrm{Y}, \mathrm{E})$, построенный на дугах Е сети $\mathrm{T}(\mathrm{M})$, по которым протекает поток величины 1. Выделено одно совершенное паросочетание

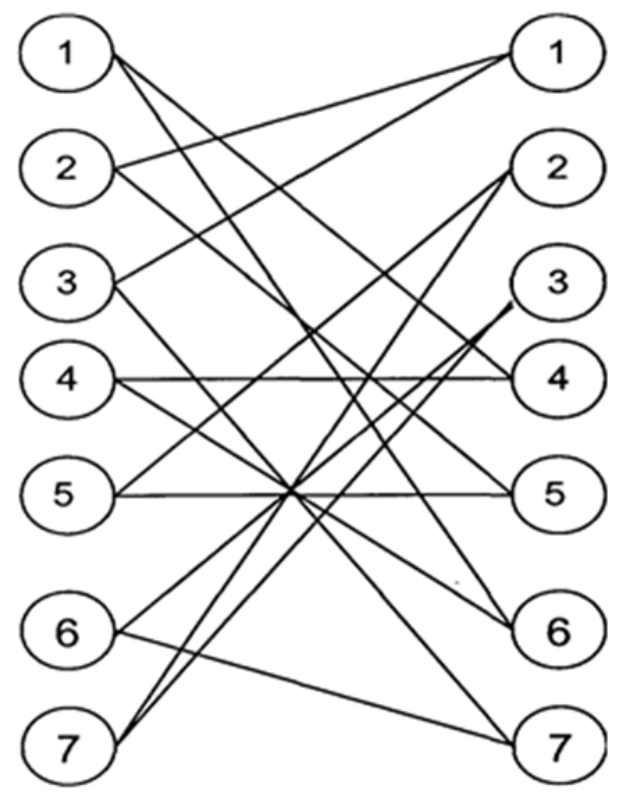

Рис. 5. Остовной подграф степени 2 результат удаления одного совершенного паросочетания из графа G, изображенного на рис. 2

Элементы множеств $X_{1}, X_{2}, X_{3}$ разместим соответственно в третьем, четвертом, пятом, элементы множеств $W_{1}, W_{2}$ - в первом, втором, шестом и седьмом столбцах предварительно обнуленной таблицы тех же размеров таким образом, чтобы каждый элемент вышеперечисленных множеств оказался в строке с тем же номером, что и в матрице $M$, и при этом все элементы в каждом столбце были попарно различны. В итоге получим дефрагментированную матрицу $M^{*}$, представленную на рис. 7.

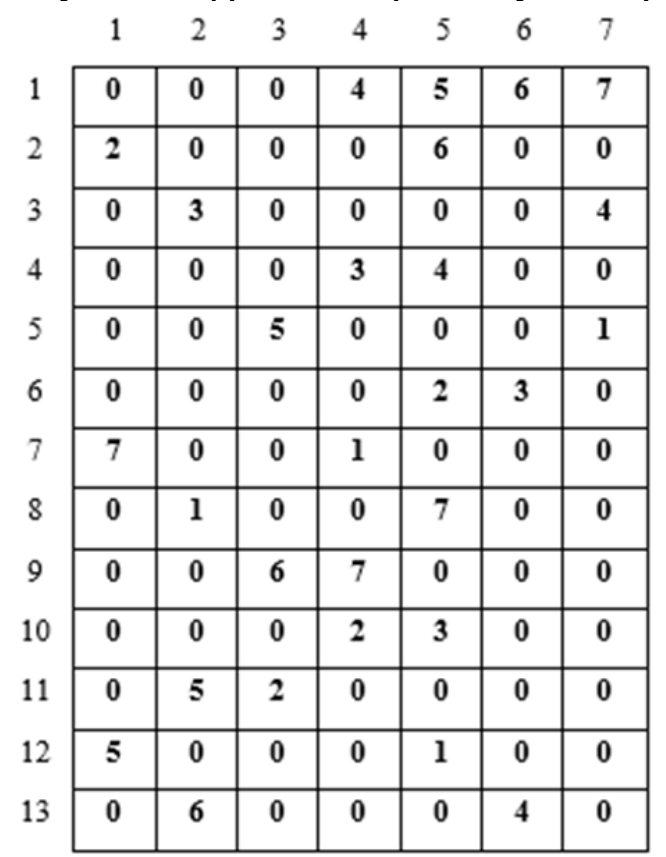

\begin{tabular}{|l|l|l|l|l|l|l|l|}
1 & 4 & 5 & 1 & 3 & 2 & 6 & 7 \\
\cline { 2 - 8 } 2 & 6 & 2 & 4 & 5 & 7 & 0 & 0 \\
\cline { 2 - 8 } 3 & 3 & 4 & 0 & 0 & 0 & 0 & 0 \\
\cline { 2 - 8 } 4 & 0 & 0 & 2 & 7 & 6 & 3 & 4 \\
\cline { 2 - 8 } 5 & 5 & 1 & 0 & 0 & 0 & 0 & 0 \\
\hline 6 & 2 & 3 & 6 & 1 & 4 & 0 & 0 \\
\hline 7 & 1 & 7 & 0 & 0 & 0 & 0 & 0 \\
\hline & 0 & 0 & 3 & 2 & 5 & 7 & 1 \\
\hline 9 & 7 & 6 & 0 & 0 & 0 & 0 & 0 \\
\cline { 2 - 8 } 10 & 0 & 0 & 5 & 4 & 1 & 2 & 3 \\
\cline { 2 - 8 } 11 & 0 & 0 & 0 & 0 & 0 & 5 & 2 \\
\cline { 2 - 8 } 12 & 0 & 0 & 7 & 6 & 3 & 1 & 5 \\
\cline { 2 - 8 } 13 & 0 & 0 & 0 & 0 & 0 & 4 & 6 \\
\hline
\end{tabular}

Рис. 6. Каждая строка матрицы $\mathrm{M}^{\prime}$ содержит Рис. 7. Дефрагментированная матрица $M^{*}$ два или четыре ненулевых элемента

Серия 1. Естественные науки. 2018. Том 33. Вып. 3 


\section{Заключение}

Помимо задачи оптимизации расписания учебных занятий, к задаче дефрагментации сводятся следующие задачи.

Задача 1 (Оптимизация размещения резидентных программ в памяти). Обозначим через $n$ количество программ, которые должны расположиться в памяти компьютера. Даны также их неделимые весовые характеристики: (объем) $a_{i}(i=1,2, \ldots, n), m$ - число блоков памяти (сокращенно $U M B), b_{j}(\mathrm{j}=1,2, \ldots, \mathrm{m})$ - объем j-го $U M B$.

В множестве программ $A$ необходимо выделить семейство $B$, которое состоит из $m$ непересекающихся подмножеств: 1) сумма весов элементов, распределенных в ј-й $\mathrm{UMB}$, не превышает $b_{j}$; 2) общая сумма весов элементов подмножества $B$ является наибольшей из возможных.

Задача 2 (Расписание для многопроцессорных машин). Необходимо выяснить существование такого расписания обработки данных, которое удовлетворяло бы некоторому заданному директивному сроку для заданного числа заданий, их длительности и количеству процессоров.

Задача 3 (Компьютерная сеть). Имеется компьютерная сеть из т каналов. В каждый квант времени по каждому каналу передается 0 или 1 сообщение. Необходимо составить такой график отправки данных по сети, чтобы сообщения шли сплошным потоком.

Примечание. С одним теоретико-графовым подходом к задаче оптимизации расписания учебных занятий можно ознакомиться в $[12,13]$.

\section{Литература}

1. Ахо А., Ульман Д., Хопкрофт Д. Построение и анализ вычислительных алгоритмов. - М.: Мир, 1979. - 536 с.

2. Гэрри М., Джонсон Д. Вычислительные машины и труднорешаемые задачи. - М.: Мир, 1982. - 416 с.

3. Ope O. Теория графов. - М.: Физматгиз, 1968. - 352 с.

4. Пападимитриу X., Стайглии К. Комбинаторная оптимизация. Алгоритмы и сложность. - М.: Мир, 1985. - 510 с.

5. Форд Л.Р., Фалкерсон Д.Р. Потоки в сетях. - М.: Мир, 1963. - 216 с.

6. $X y$ T. Целочисленное программирование и потоки в сетях. - М.: Мир, 1974. $520 \mathrm{c}$

7. Магомедов А.М. Связность матрицы перестановок в случае четырех столбцов. Деп. в ВИНИТИ, 8700-В-87.

8. Магомедов А.М. Дефрагментация таблицы перестановок из 4 столбцов // Дискретная математика. - 2009. - Т. 21, вып. 4. - С. 95-104.

9. Якубов А.3. Дефрагментация строк матрицы перестановок. Деп. в ВИНИТИ № 1638-B2002, 2002. - $10 \mathrm{c}$.

10. Якубов А.3. Потоковые методы в решении задачи дефрагментации матрицы расписания // Материалы IV Межд. конференции «Нелокальные краевые задачи и родственные проблемы математической биологии, информатики и физики». - Нальчик: Терскол, 2013. - С. 115-117.

11. Якубов А.3. Моделирование безоконного расписания потоковыми методами // Материалы XII Международной научной конференции «Порядковый анализ и смежные вопросы математического моделирования» / Владикавказский научный центр Российской академии наук, Южный математический институт, Северо-Осетинский государственный университет имени К.Л. Хетагурова, Южный федеральный университет. - 
Владикавказ: ЮМИ ВНЦ РАН, 2015. - С. 239-240.

12. Магомедов А.М. Магомедов Т.А. Реберно-вершинные инцидентные паросочетания в задачах расписаний // Прикладная дискретная математика. - 2015. - № 1 (27). - C. 92-95.

13. Магомедов А.М., Магомедов Т.А. Последовательное разбиение ребер двудольного графа на паросочетания // Дискретная математика. - 2016. - Т. 28, вып. 1. C. $78-86$.

Поступила в редакциию 7 сентября 2018 г.

UDC 519.618

DOI: $10.21779 / 2542-0321-2018-33-3-73-79$

\title{
The use of flow graph methods in scheduling optimization
}

\begin{abstract}
A.Z. Yakubov
Dagestan State University; Russia, 367001, Makhachkala, M. Gadzhiev st., 43a; yakubovaz@mail.ru

The task of optimizing the curriculum is formulated in the form of a matrix defragmentation. There is the existence of seven columns matrix, each of which contains, in addition to zeros, some permutation of the first $\mathrm{n}$ natural numbers, a matrix of the same dimensions that preserves the set of elements of the original matrix in each column and each row, and thus in each row all non-zero elements are located together. Due to the use of streaming graph methods the necessary and sufficient conditions of matrix defragmentation in some special cases are found.
\end{abstract}

Keywords: scheduling optimization, defragmentation of matrix, streams in networks, matching.

Received 7 September, 2018 